\title{
Evaluation of soil carbon simulation in CMIP6 Earth System Models
}

Rebecca M. Varney ${ }^{1}$, Sarah E. Chadburn ${ }^{1}$, Eleanor J. Burke ${ }^{2}$, and Peter M. Cox ${ }^{1}$

${ }^{1}$ College of Engineering, Mathematics and Physical Sciences, University of Exeter, Laver Building, North Park Road, Exeter, EX4 $4 Q F, U K$

${ }^{2}$ Met Office Hadley Centre, FitzRoy Road, Exeter, EX1 3PB, UK

Correspondence: Rebecca M. Varney (r.varney@exeter.ac.uk)

Supplementary Material 
ACCESS-ESM1-5

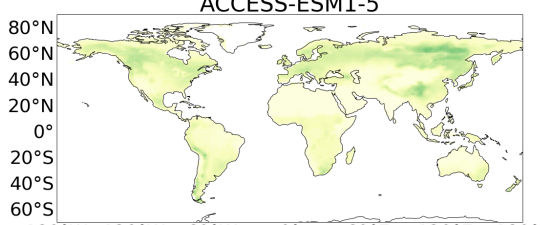

$180^{\circ} \mathrm{W} \quad 120^{\circ} \mathrm{W} \quad 60^{\circ} \mathrm{W} \quad 0^{\circ} \quad 60^{\circ} \mathrm{E} \quad 120^{\circ} \mathrm{E} \quad 180^{\circ} \mathrm{E} \quad 180^{\circ}$

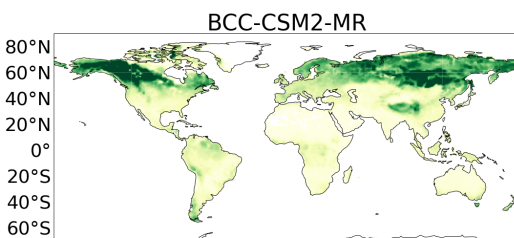

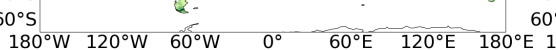
CanESM5
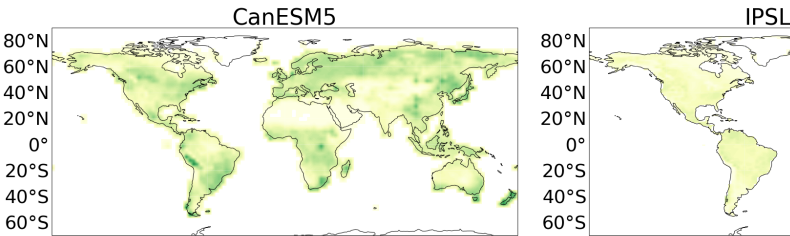

IPSL-CM6A-LR
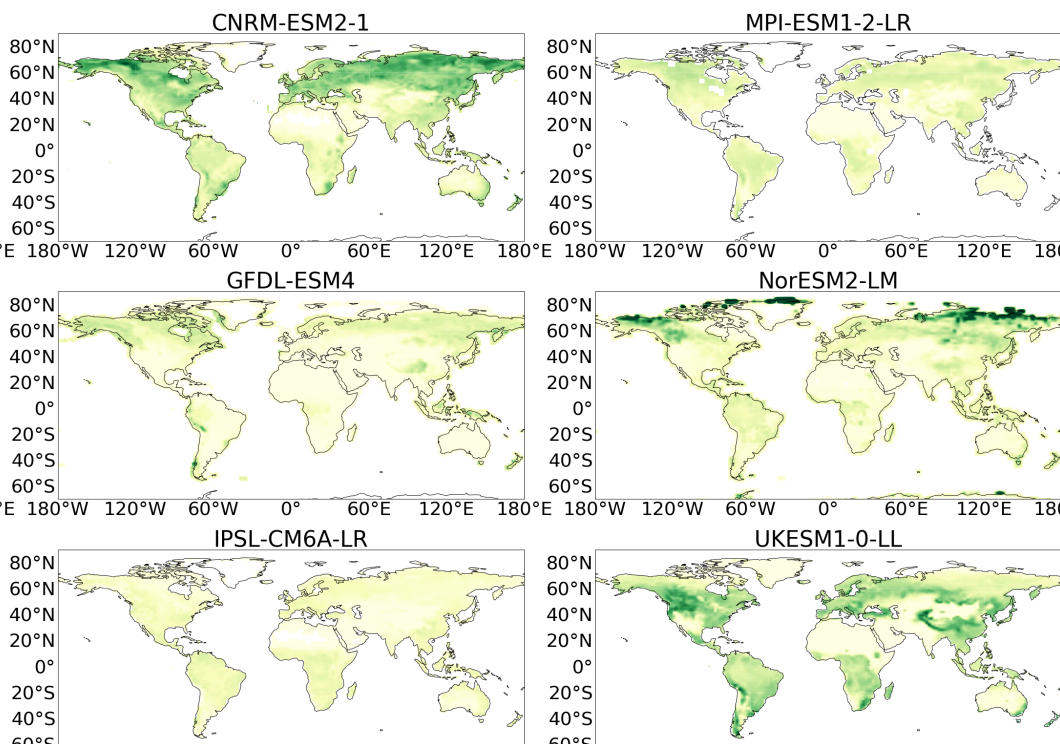

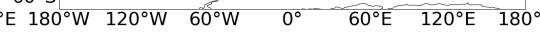
NorESM2-LM

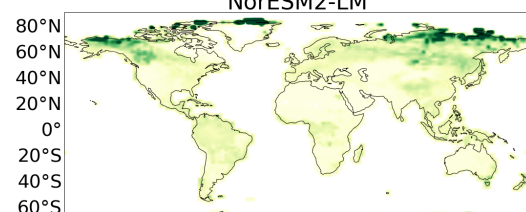

$60^{\circ} \mathrm{S}$

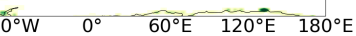
UKESM1-0-LL

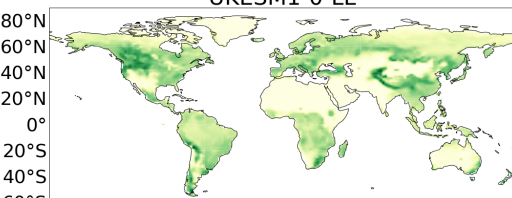

$60^{\circ} \mathrm{S}$ MIROC-ES2L

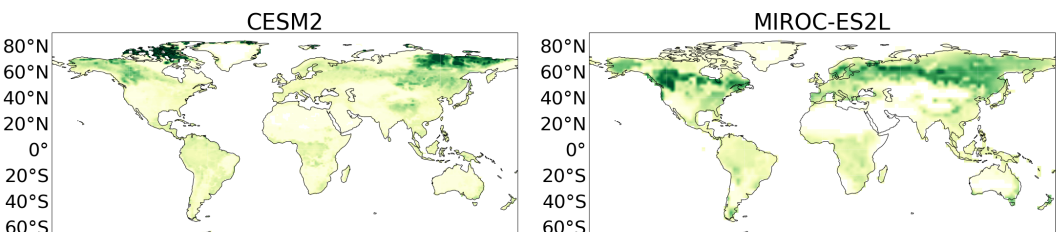

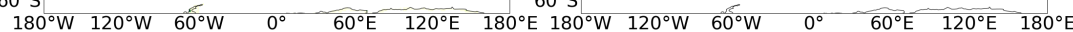

Figure S1. Maps of soil carbon $\left(C_{s}\right)$ in the historical simulation (1950-2000) for the CMIP6 models.
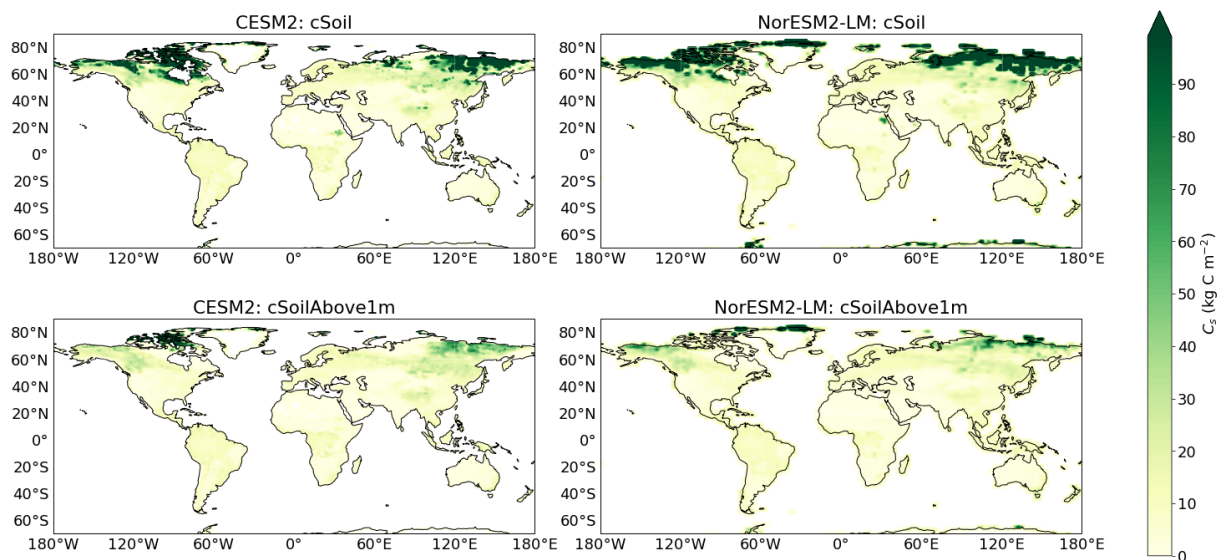

Figure S2. Maps comparing the soil carbon $\left(C_{s}\right)$ output variables $c$ Soil and $c$ SoilAbove $1 m$ in the historical simualtion (1950-2000) of the CMIP6 models CESM2 and NorESM2-LM. 
BNU-ESM

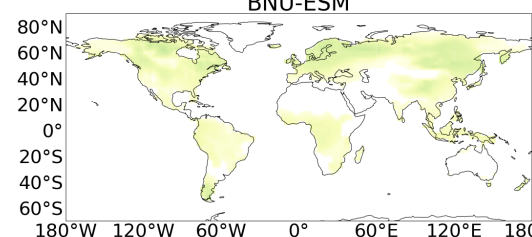

CCSM4

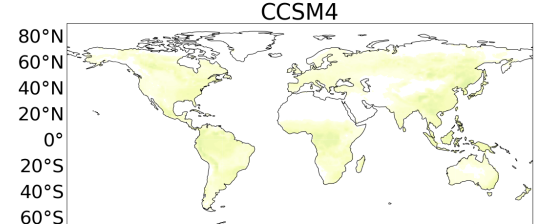

GISS-E2-R

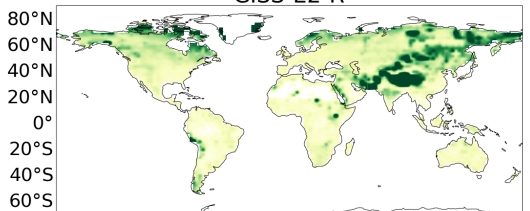

MPI-ESM-LR

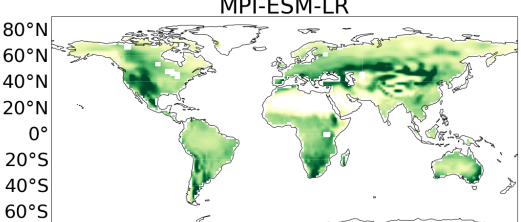

HadGEM2-ES

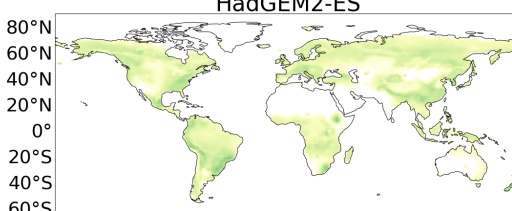

NorESM1-M

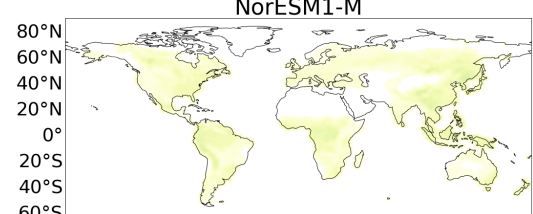

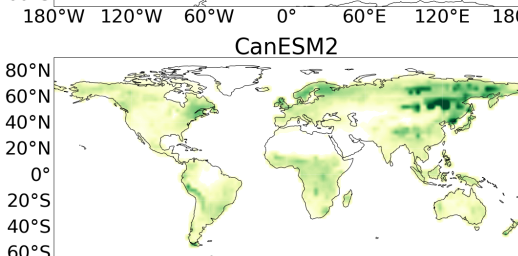

NESM2

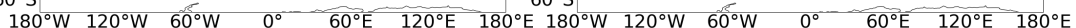
GFDL-ESM2G

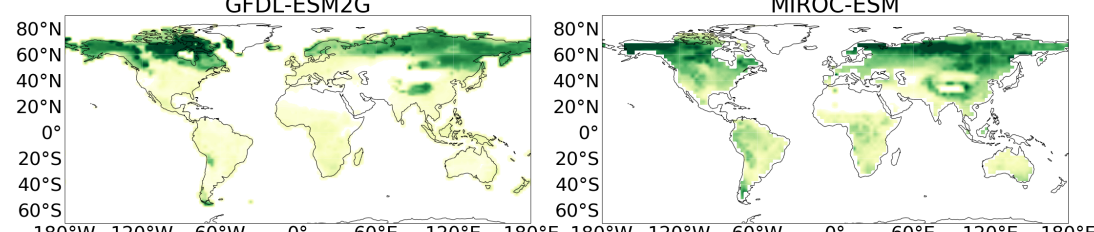

IPSL-CM5A-LR

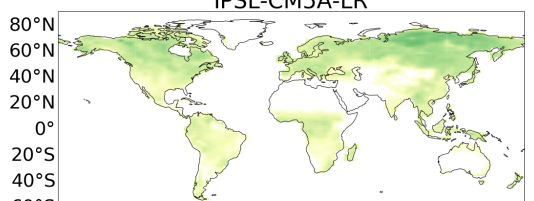

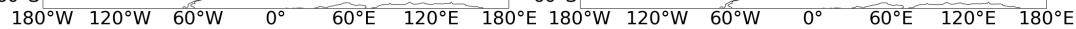

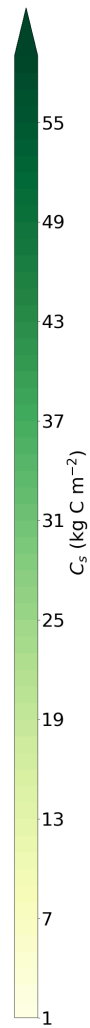

Figure S3. Maps of soil carbon $\left(C_{s}\right)$ in the historical simulation (1950-2000) for the CMIP5 models. 
ACCESS-ESM1-5

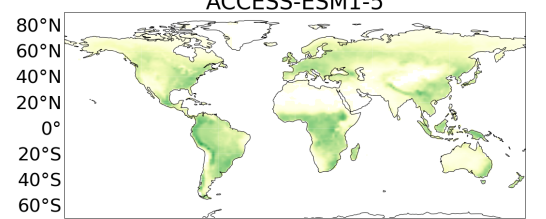

$60^{\circ} \mathrm{S}$

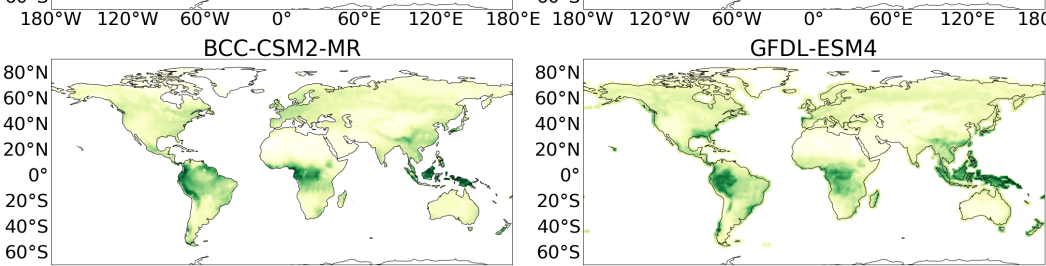

BCC-CSM2-MR

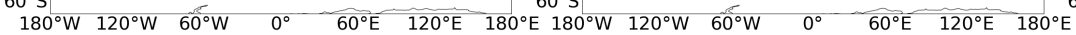
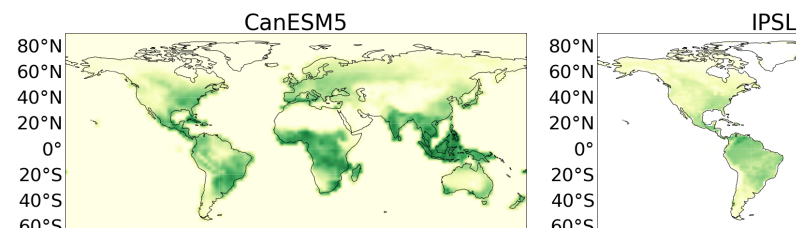

IPSL-CM6A-LR
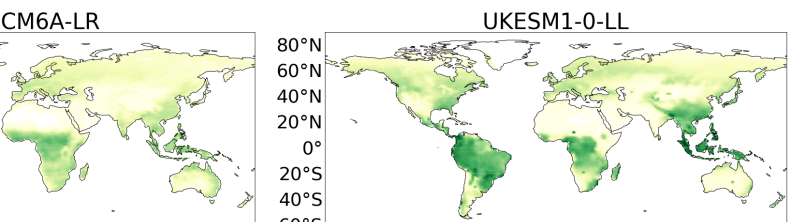

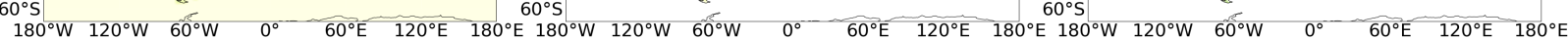
MIROC-ES2L

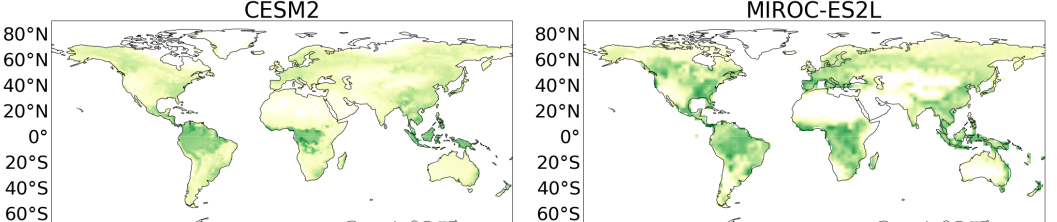

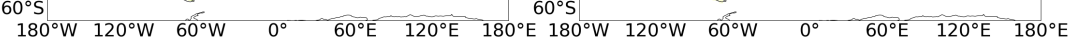
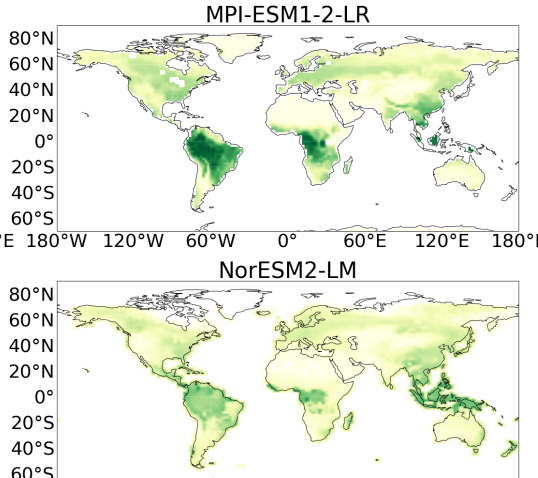

NorESM2-LM

$0^{\circ} \mathrm{W} \quad 0^{\circ} \quad 60^{\circ} \mathrm{E} \quad 120^{\circ} \mathrm{E} \quad 180^{\circ} \mathrm{E}$

Figure S4. Maps of Net Primary Productivity (NPP) in the historical simulation (1995-2005) for the CMIP6 models. 

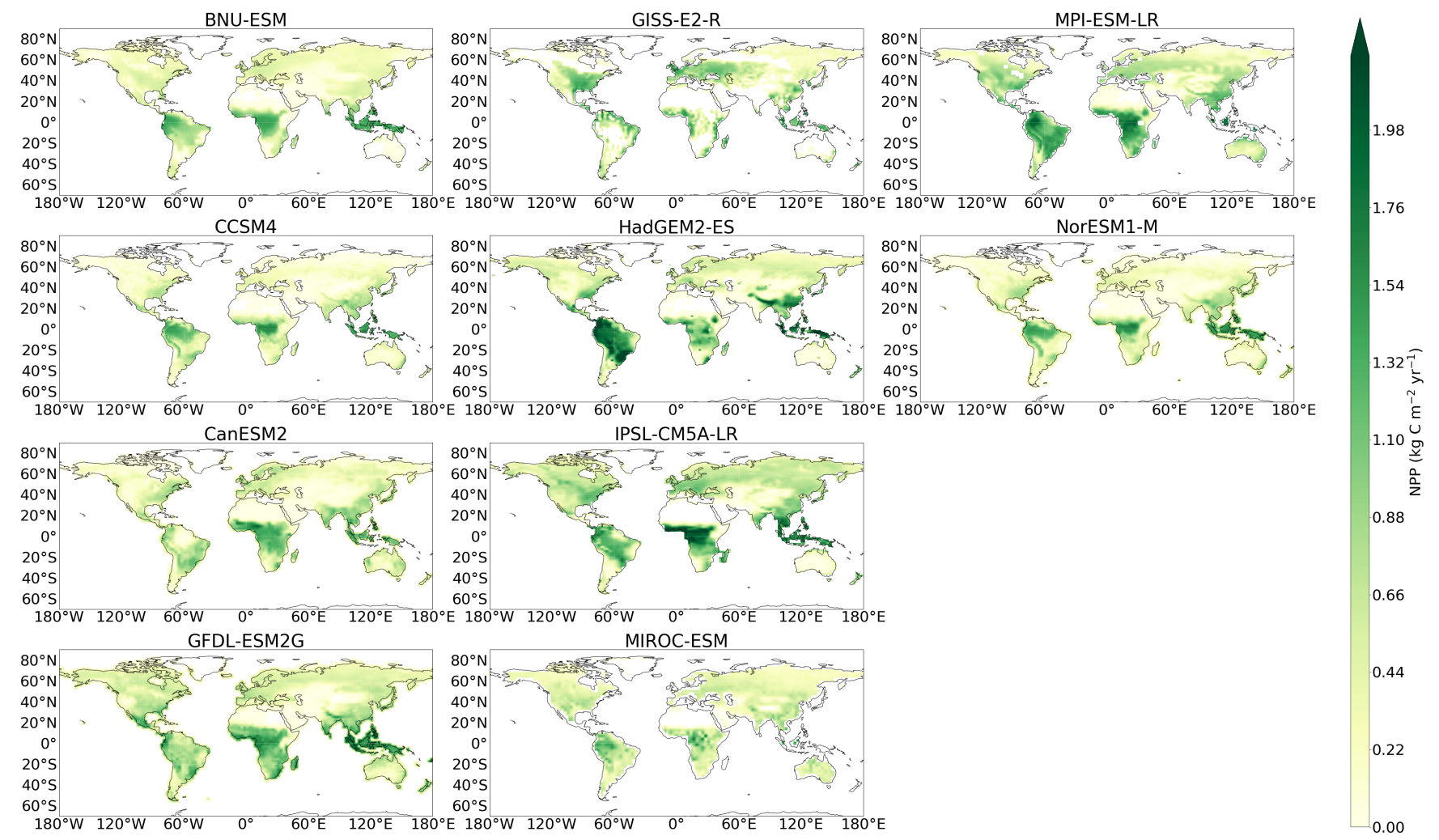

Figure S5. Maps of Net Primary Productivity (NPP) in the historical simulation (1995-2005) for the CMIP5 models. 
ACCESS-ESM1-5

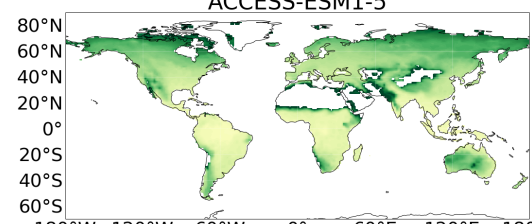

$180^{\circ} \mathrm{W} \quad 120^{\circ} \mathrm{W} \quad 60^{\circ} \mathrm{W} \quad 0^{\circ} \quad 60^{\circ} \mathrm{E} \quad 120^{\circ} \mathrm{E} \quad 180^{\circ} \mathrm{E}$ BCC-CSM2-MR

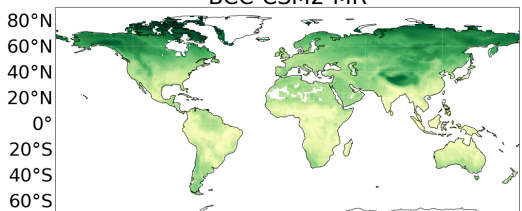

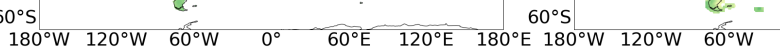
CanESM5
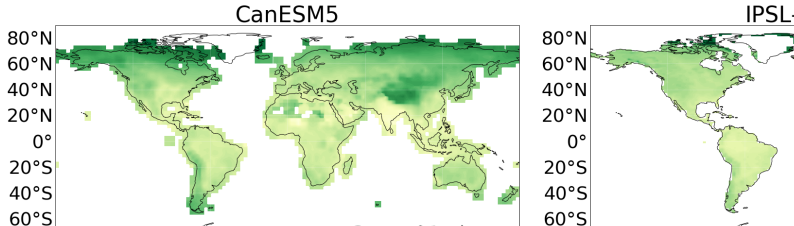

IPSL-CM6A-LR
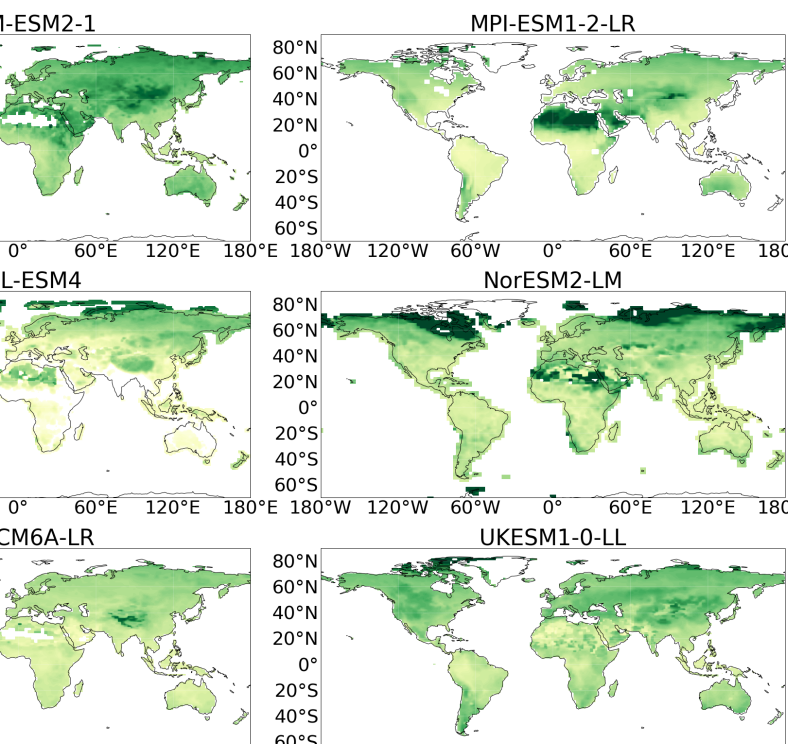

$60^{\circ} \mathrm{S}$

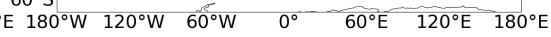
NorESM2-LM
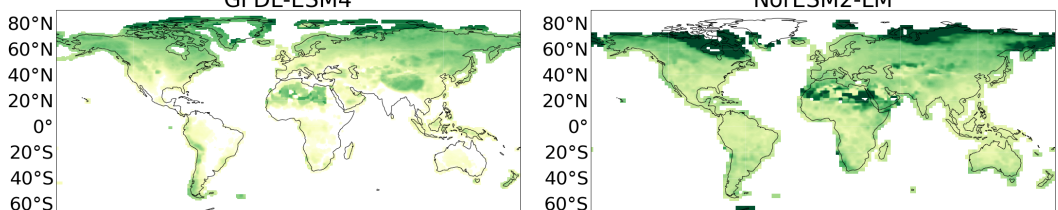

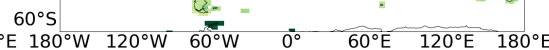

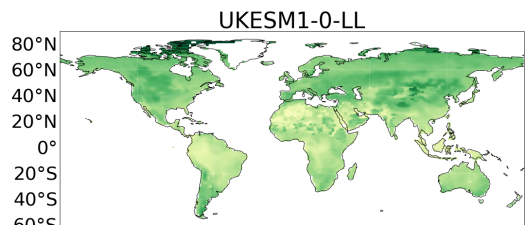

UKESM1-0-LL MIROC-ES2L

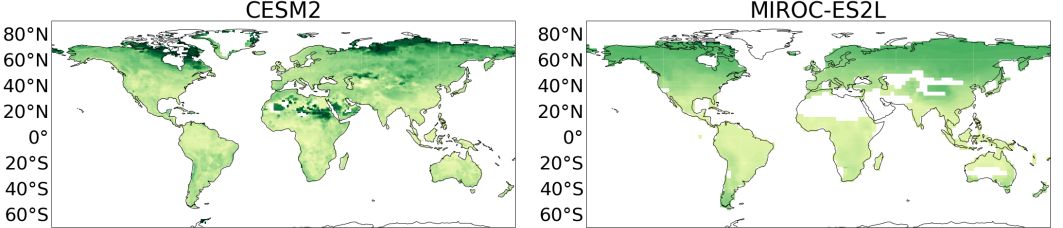

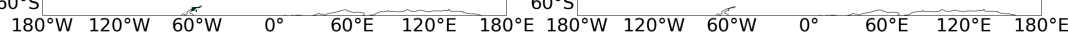

Figure S6. Maps of soil carbon turnover times $\left(\tau_{s}\right)$ in the historical simulation for the CMIP6 models, where $\tau_{s}$ is defined as the ratio of $C_{s}$ (1950-2000) to $R_{h}$ (1995-2005). 
BNU-ESM

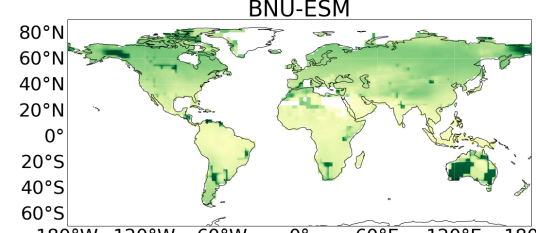

GISS-E2-R
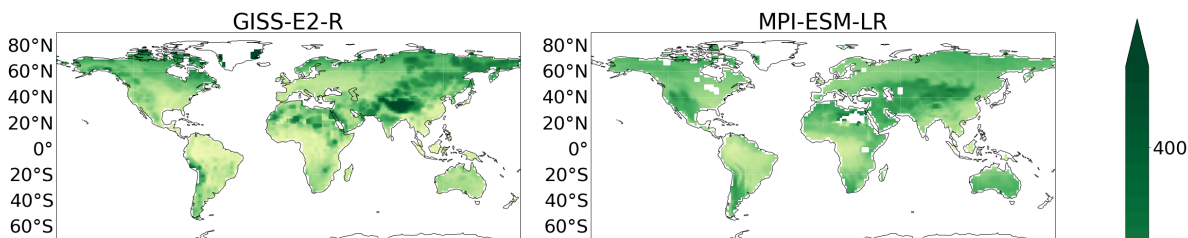

CCSM4

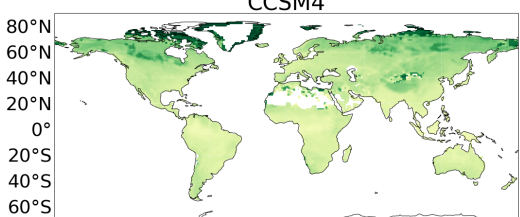
HadGEM2-ES
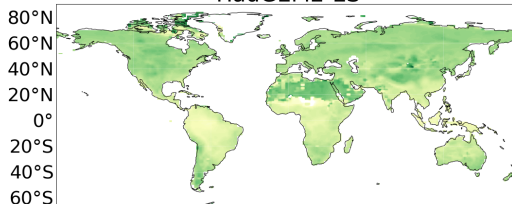

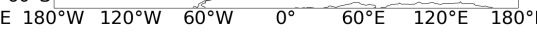
NorESM1-M

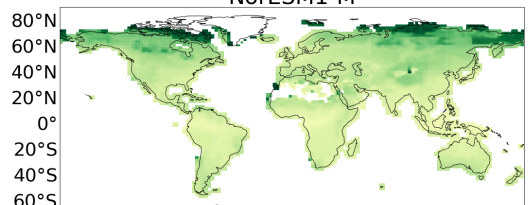

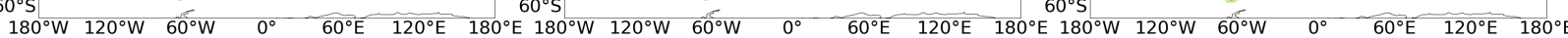
CanESM2

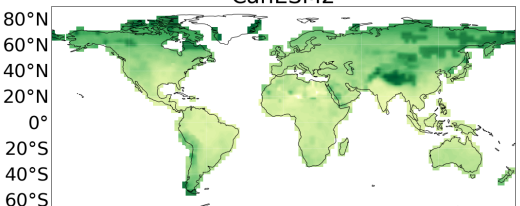

IPSL-CM5A-LR

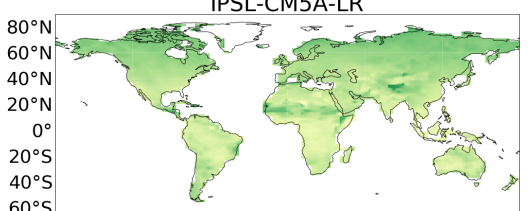

MIROC-ESM

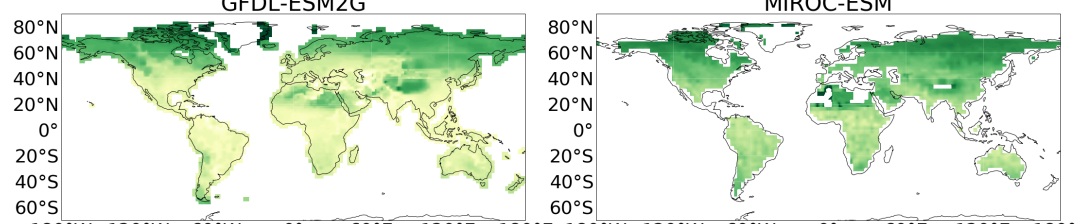

$180^{\circ} \mathrm{W} 120^{\circ} \mathrm{W} \quad 60^{\circ} \mathrm{W} \quad 0^{\circ} 60^{\circ} \mathrm{E} \quad 120^{\circ} \mathrm{E} \quad 180^{\circ} \mathrm{E} 180^{\circ} \mathrm{W} 120^{\circ} \mathrm{W} \quad 60^{\circ} \mathrm{W} \quad 0^{\circ} \quad 60^{\circ} \mathrm{E} \quad 120^{\circ} \mathrm{E} \quad 180^{\circ} \mathrm{E}$

Figure S7. Maps of soil carbon turnover times $\left(\tau_{s}\right)$ in the historical simulation for the CMIP5 models, where $\tau_{s}$ is defined as the ratio of $C_{s}$ (1950-2000) to $R_{h}(1995-2005)$. 


\begin{tabular}{|c|c|c|c|c|c|c|c|c|c|c|c|c|c|}
\hline 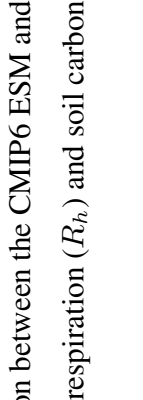 & $\sum_{\Omega}^{\mathscr{N}}$ & 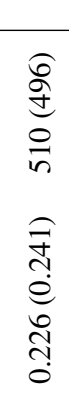 & 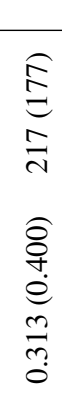 & 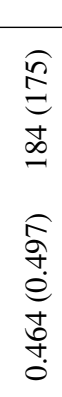 & 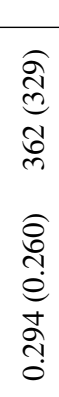 & 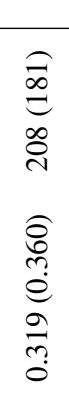 & 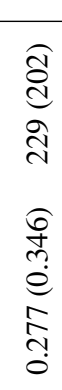 & 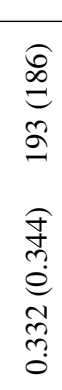 & 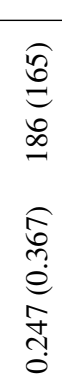 & 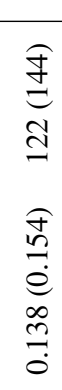 & 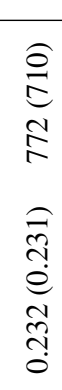 & 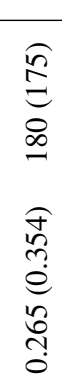 & 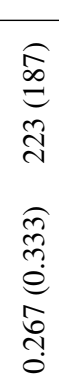 \\
\hline 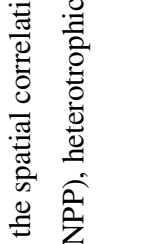 & 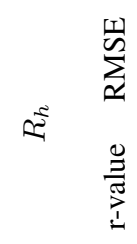 & $\begin{array}{l}\text { J } \\
0 \\
\overline{0} \\
\stackrel{0}{0}\end{array}$ & $\begin{array}{l}\stackrel{3}{ \pm} \\
\vdots \\
\frac{2}{0} \\
0\end{array}$ & $\begin{array}{l}\stackrel{2}{f} \\
\stackrel{0}{0} \\
\infty \\
0 \\
0 \\
0\end{array}$ & 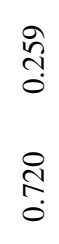 & $\begin{array}{l}\text { ڤ్రి } \\
0 \\
\text { aे } \\
\stackrel{0}{0}\end{array}$ & 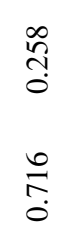 & 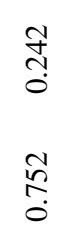 & $\begin{array}{l}\mathscr{\infty} \\
\stackrel{0}{0} \\
\overrightarrow{0} \\
\stackrel{0}{0}\end{array}$ & 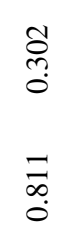 & 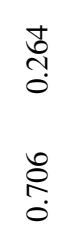 & $\begin{array}{l}\frac{a}{n} \\
\vdots \\
\frac{8}{0} \\
\stackrel{0}{0}\end{array}$ & $\begin{array}{l}\frac{n}{n} \\
0 \\
0 \\
0 \\
0\end{array}$ \\
\hline 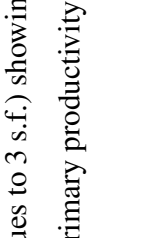 & 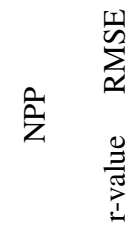 & 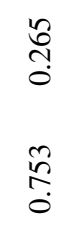 & 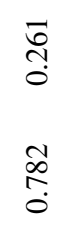 & 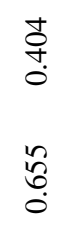 & 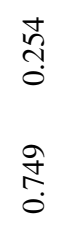 & $\begin{array}{l}\stackrel{n}{+} \\
\stackrel{0}{0} \\
\stackrel{0}{\infty} \\
\stackrel{\infty}{0}\end{array}$ & 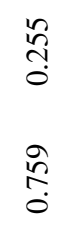 & $\begin{array}{l}\tilde{N} \\
\tilde{0} \\
\stackrel{0}{0} \\
\stackrel{0}{0}\end{array}$ & 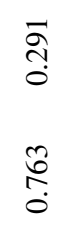 & 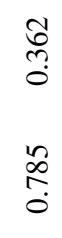 & 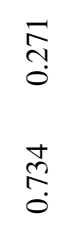 & $\begin{array}{l}n \\
0 \\
0 \\
0 \\
0 \\
0 \\
0 \\
0\end{array}$ & $\begin{array}{l}\stackrel{8}{0} \\
0 \\
0 \\
\infty \\
0 \\
0\end{array}$ \\
\hline 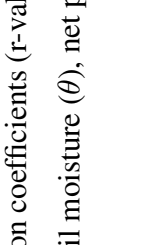 & 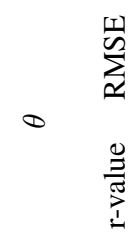 & 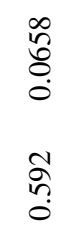 & $\begin{array}{l}8 \\
8 \\
0 \\
0 \\
\hat{0} \\
0 \\
0\end{array}$ & $\begin{array}{l}= \\
0 \\
0 \\
0 \\
\stackrel{0}{0} \\
0 \\
0\end{array}$ & 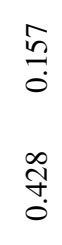 & $\begin{array}{l}8 \\
0 \\
0 \\
0 \\
0 \\
1 \\
0 \\
0 \\
0\end{array}$ & 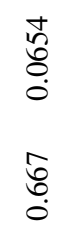 & 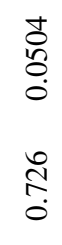 & $\begin{array}{l}\hat{\sigma} \\
\hat{0} \\
\dot{0} \\
\hat{0} \\
0 \\
0\end{array}$ & $\begin{array}{l}\tilde{8} \\
8 \\
0 \\
0 \\
\delta \\
0 \\
0 \\
0\end{array}$ & 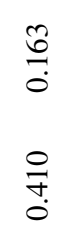 & \begin{tabular}{l}
\multirow{7}{*}{} \\
0 \\
0 \\
0 \\
0 \\
0 \\
0 \\
0
\end{tabular} & $\begin{array}{l}+ \\
\hat{a} \\
\dot{0} \\
0 \\
0 \\
\dot{0} \\
0\end{array}$ \\
\hline 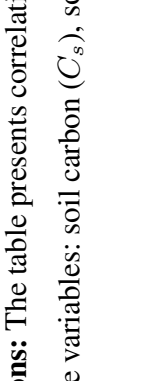 & $\sum_{\Omega}^{\infty}$ & 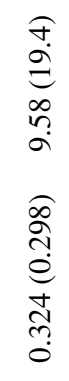 & 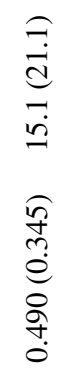 & 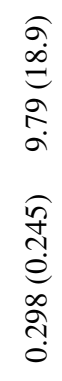 & 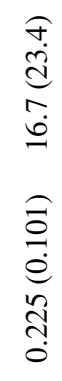 & 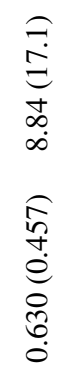 & 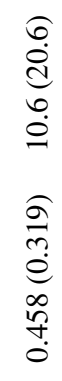 & 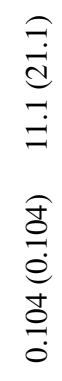 & 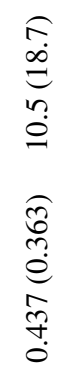 & 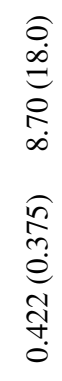 & 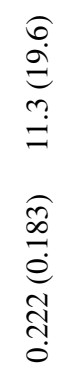 & 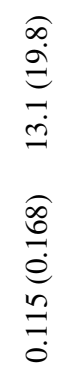 & 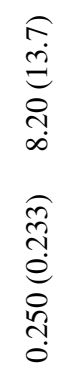 \\
\hline 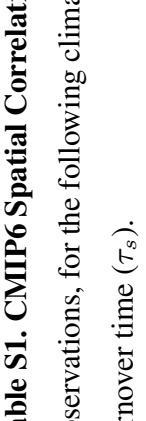 & 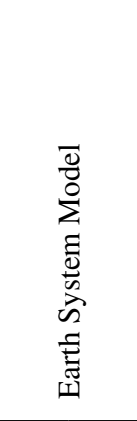 & 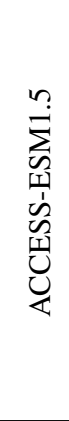 & 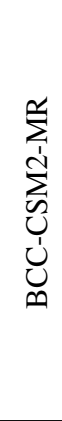 & 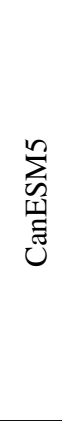 & $\begin{array}{l}\sum_{i=1}^{̃} \\
\text { In }\end{array}$ & $\begin{array}{l}\sum_{n=1}^{1} \\
\sum_{11}^{1} \\
\underbrace{1}_{U}\end{array}$ & 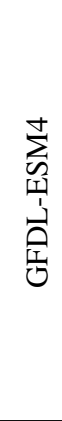 & $\begin{array}{l}\frac{a}{1} \\
\sum_{0}^{1} \\
\sum_{0}^{0} \\
\frac{1}{2} \\
0\end{array}$ & 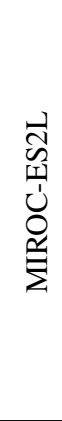 & 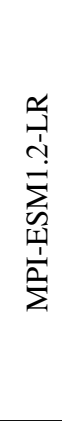 & 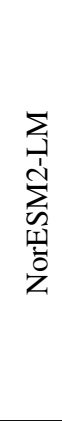 & 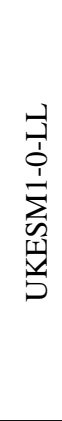 & 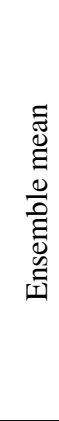 \\
\hline
\end{tabular}




\begin{tabular}{|c|c|c|c|c|c|c|c|c|c|c|c|c|}
\hline 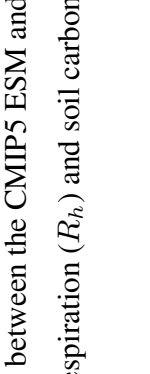 & 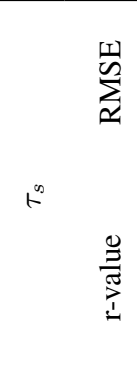 & 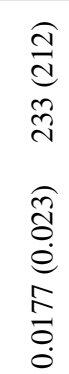 & 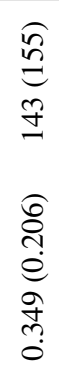 & 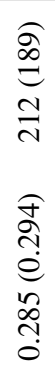 & 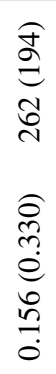 & 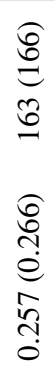 & 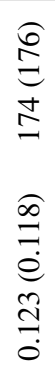 & 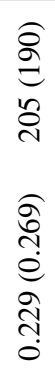 & 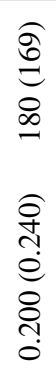 & 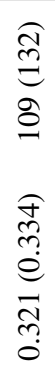 & 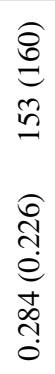 & 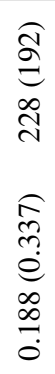 \\
\hline 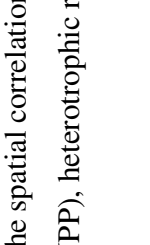 & 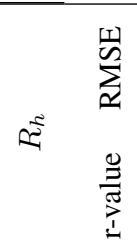 & \begin{tabular}{l}
$\stackrel{શ}{\hat{0}}$ \\
\multirow{0}{0}{} \\
$\stackrel{2}{0}$ \\
0
\end{tabular} & $\stackrel{\text { N }}{\text { ป̦ }}$ & 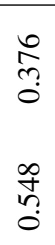 & $\begin{array}{l}\stackrel{2}{?} \\
\text { ?. } \\
\frac{\infty}{6} \\
\stackrel{0}{0}\end{array}$ & 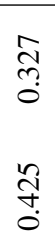 & $\begin{array}{l}\stackrel{0}{+} \\
\stackrel{0}{0} \\
\stackrel{+}{\stackrel{J}{0}}\end{array}$ & 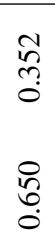 & 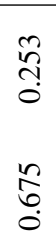 & $\begin{array}{l}\overline{7} \\
\stackrel{0}{0} \\
\stackrel{8}{0} \\
\stackrel{0}{0}\end{array}$ & 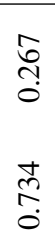 & $\begin{array}{l}\frac{2}{0} \\
\stackrel{0}{0} \\
\stackrel{8}{0}\end{array}$ \\
\hline 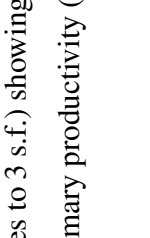 & 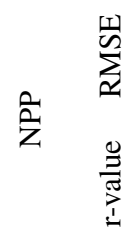 & $\begin{array}{l}\frac{\infty}{0} \\
0 \\
\tilde{0} \\
\tilde{0} \\
0\end{array}$ & $\begin{array}{l}\stackrel{+}{\infty} \\
\stackrel{1}{0} \\
\stackrel{n}{2} \\
\stackrel{0}{0}\end{array}$ & 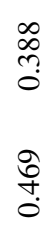 & 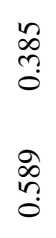 & 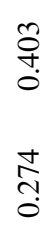 & \begin{tabular}{l}
$\stackrel{2}{2}$ \\
$\stackrel{5}{0}$ \\
\multirow{0}{0}{} \\
$\stackrel{0}{0}$
\end{tabular} & 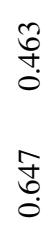 & 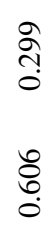 & 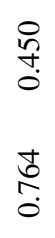 & $\begin{array}{l}\hat{\jmath} \\
\tilde{0} \\
\frac{1}{0} \\
0\end{array}$ & 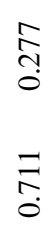 \\
\hline 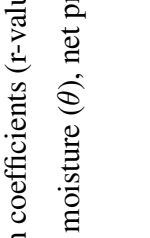 & 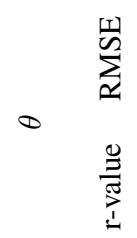 & 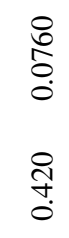 & 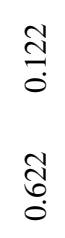 & $\begin{array}{l}n \\
2 \\
\infty \\
0 \\
0 \\
\\
\infty \\
n \\
0\end{array}$ & 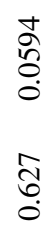 & 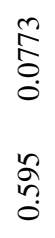 & 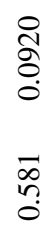 & $\begin{array}{l}0 \\
\stackrel{0}{0} \\
\stackrel{\tilde{n}}{n} \\
\stackrel{0}{0}\end{array}$ & $\begin{array}{l}\stackrel{0}{+} \\
\stackrel{+}{0} \\
0 \\
0\end{array}$ & , & 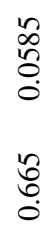 & 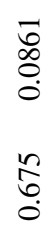 \\
\hline 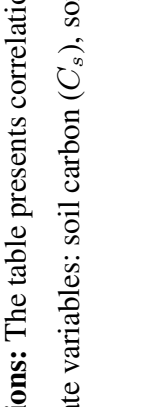 & 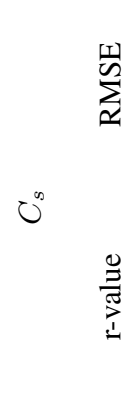 & 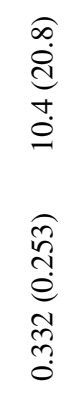 & 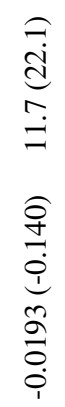 & 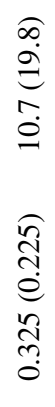 & 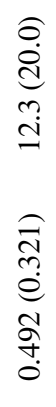 & 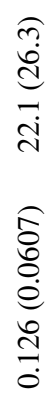 & 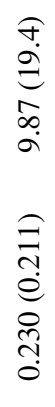 & 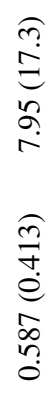 & 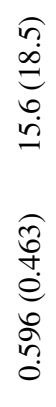 & 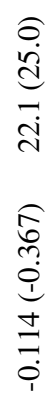 & 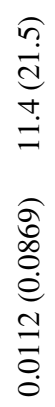 & 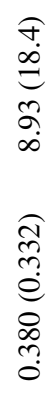 \\
\hline 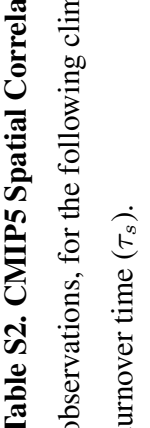 & 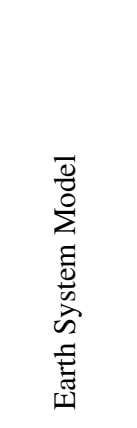 & 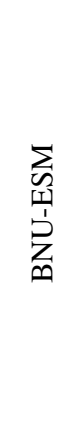 & $\sum_{0}^{+D}$ & 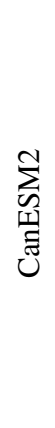 & 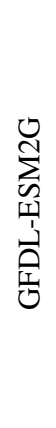 & 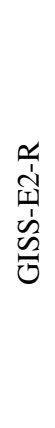 & 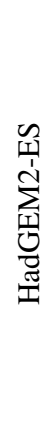 & 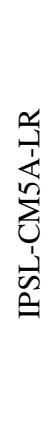 & 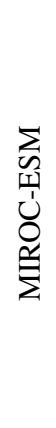 & 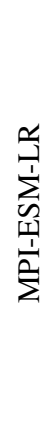 & 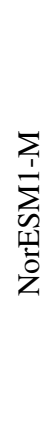 & 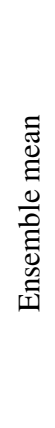 \\
\hline
\end{tabular}


Table S3. CMIP6 Spatial Correlations: The table shows r-values representing the spatial correlation between soil carbon $\left(C_{s}\right)$ and climate variables (NPP, soil moisture and temperature) from each ESM and the observations. EXTRA NPP ONES. The table shows r-values representing the spatial correlation between soil carbon turnover time $\left(\tau_{s}\right)$ and climate variables (soil carbon, NPP, soil moisture and temperature) from each ESM and the observations. All rounded to 3 significant figures.

\begin{tabular}{|c|c|cc|c|cc|}
\hline Earth System Model & $C_{s}$-NPP & NPP- $\theta$ & NPP-T & $C_{s}-\tau_{s}$ & $\tau_{s}-\theta$ & $\tau_{s}-\mathrm{T}$ \\
\hline ACCESS-ESM1.5 & 0.644 & -0.288 & 0.566 & -0.181 & -0.0871 & -0.0872 \\
\hline BCC-CSM2-MR & 0.214 & -0.329 & 0.543 & 0.129 & 0.229 & -0.368 \\
\hline CanESM5 & 0.789 & 0.0423 & 0.301 & -0.216 & 0.430 & -0.781 \\
\hline CESM2 (cSoilAbove1m) & 0.134 & 0.0211 & 0.549 & -0.00142 & 0.242 & -0.266 \\
\hline CESM2 (cSoil) & -0.00720 & - & - & 0.214 & 0.335 & -0.287 \\
\hline CNRM-ESM2-1 & 0.645 & 0.0102 & 0.594 & 0.0188 & -0.0533 & -0.314 \\
\hline GFDL-ESM4 & 0.573 & 0.697 & 0.196 & -0.100 & 0.0420 & -0.537 \\
\hline IPSL-CM6A-LR & 0.871 & 0.333 & 0.541 & -0.219 & 0.431 & -0.431 \\
\hline MIROC-ES2L & 0.630 & 0.154 & 0.545 & 0.144 & 0.111 & -0.268 \\
\hline MPI-ESM1.2-LR & 0.704 & 0.657 & 0.548 & -0.278 & -0.319 & 0.158 \\
\hline NorESM2-LM (cSoilAbove1m) & 0.261 & 0.499 & 0.232 & 0.153 & 0.357 & -0.448 \\
\hline NorESM2-LM (cSoil) & 0.0293 & - & - & 0.308 & 0.252 & -0.317 \\
\hline UKESM1-0-LL & 0.749 & 0.587 & 0.563 & -0.0933 & 0.0618 & -0.299 \\
\hline Ensemble mean & 0.424 & 0.494 & 0.257 & -0.0605 & 0.198 & -0.398 \\
\hline Benchmark datasets & -0.0229 & 0.646 & 0.435 & 0.232 & -0.239 & -0.296 \\
\hline
\end{tabular}


Table S4. CMIP5 Spatial Correlations: The table shows r-values representing the spatial correlation between soil carbon $\left(C_{s}\right)$ and NPP from each ESM and the observations and r-values representing the spatial correlation between soil carbon $\left(C_{s}\right)$ and soil moisture $(\theta)$ from each ESM and the observations. All rounded to 3 significant figures.

\begin{tabular}{|c|c|cc|c|cc|}
\hline Earth System Model & $C_{s}$-NPP & NPP- $\theta$ & NPP-T & $C_{s}-\tau_{s}$ & $\tau_{s}-\theta$ & $\tau_{s}-\mathrm{T}$ \\
\hline BNU-ESM & 0.623 & -0.420 & 0.513 & -0.131 & 0.0351 & -0.0775 \\
\hline CCSM4 & -0.00541 & -0.348 & 0.551 & -0.181 & 0.450 & -0.301 \\
\hline CanESM2 & 0.687 & 0.0603 & 0.212 & 0.188 & 0.357 & -0.689 \\
\hline GFDL-ESM2G & 0.428 & 0.767 & 0.213 & 0.0337 & -0.0889 & -0.293 \\
\hline GISS-E2-R & -0.156 & 0.492 & -0.0113 & 0.897 & -0.0382 & -0.473 \\
\hline HadGEM2-ES & 0.776 & 0.558 & 0.529 & -0.165 & 0.0317 & -0.355 \\
\hline IPSL-CM5A-LR & 0.500 & 0.302 & 0.201 & 0.379 & 0.492 & -0.685 \\
\hline MIROC-ESM & 0.466 & 0.649 & 0.702 & 0.119 & 0.0432 & -0.236 \\
\hline MPI-ESM-LR & 0.681 & - & 0.653 & 0.0682 & - & -0.159 \\
\hline NorESM1-M & 0.898 & 0.0987 & 0.228 & -0.150 & 0.0836 & -0.242 \\
\hline Ensemble mean & 0.222 & 0.258 & 0.230 & 0.108 & 0.210 & -0.314 \\
\hline Benchmark datasets & -0.0229 & 0.646 & 0.435 & 0.232 & -0.239 & -0.296 \\
\hline
\end{tabular}

